\title{
COMPORTAMIENTO RELACIONADO CON SABIDURÍA EN ADOLESCENTES. VALIDEZ ESTRUCTURAL DE UNA ESCALA DE CALIFICACIÓN PARA PROFESORES
}

\author{
Pedro González Leandro* \\ Alicia Asef** \\ Universidad de La Laguna (Islas Canarias, España)* \\ Universidad Nacional de San Martín, Fundación del Gran Rosario (Rosario, Argentina)**
}

\section{RESUMEN}

Se presentan resultados de una escala de calificación sobre "sabiduria» cumplimentada por profesores y sobre sus alumnos de enseñanza secundaria ( $N=164)$ en Árgentina. Se presentan resultados de composición factorial y fiabilidad de la escala que aisla dos factores claros (relacionados entre si). También se enumeran algunos problemas y limitaciones del instrumento, $y$ se plantean posibles estudios futuros, dirigidos al perfeccionamiento de la escala y a posibles aplicaciones de la misma.

Palabras clave: SABIDURIA EN ADOLESCENTES, EVALUACIÓN, ESCALAS DE CALIFCACIÓN.

\section{SUMMARY}

A rating scale on the assessment of adolescents behaviour related to wisdom is shown (15 items, 164 subjects). The scale was fulfilled by the teachers. The items have been proposed

Correspondencia: Pedro González Leandro, Facultad de Psicologia, Universidad de La Laguna. Campus de Guajara, 38205 La Laguna (Islas Canarias - España). Correo electrónico: pleandro@ull.es. 
from three theories of wisdom (Baltes, Stemberg, Ardelt). A factor structure of two factors have been obtained and the reliability coefficients were satisfactory. Some problems and limitations of the instrument are enumerated, and improvements of the scale for future studies and its applications are discussed.

Koy words: MSDOM IN ADOLESCENCE, ASSESSMENT, RATNG SCALES.

La sabiduría es un concepto antiguo que desde siempre ha estado ligado a la filosofla y a la religión, y se puede decir que es a raíz de la propuesta de Erikson (Erikson, 1959, 1968)' ${ }^{1}$ sobre el desarrollo humano, que situaba la aparición de la sabiduría en el último estadio de la vida, cuando los psicólogos empiezan a interesarse por su estudio como un constructo psicológico. Sin embargo, el escaso apoyo empírico que ha tenido la teorla de los estadios, unido a que Erikson no aportó una definición precisa del constructo sabiduría, hace que su propuesta sea difícil de evaluarse empíricamente. Además, como apuntan Clayton y Birren (1980), el enfoque de Erikson se centra en el desarrollo socio-emocional y nada dice del papel que juegan, en el desarrollo psicosocial de la persona, los componentes reflexivos (introspección e intuición) e intelectuales de la sabiduría.

Csikszentmihalyi y Rathunde (1990), desde un punto de vista de hermenéutica evolucionista aplicada al concepto de sabiduria, muestran como a lo largo de la historia se han mantenido ciertas continuidades en el significado del término sabiduria; que estas continuidades representan prerrequisitos funcionales, que han cambiado muy poco a lo largo del tiempo, y que se refieren al

\footnotetext{
1 Para Erikson la sabidurla es una virtud que emerge en el último estadio de la vida (el octavo) como superación de la oposiclón: Integrldad del yo versus desesperación; la persona sabia serla la que, aparte de comprender el significado de su vida y de la exlatencla en general, posee el equilibrio necesario para afrontar el hecho de su propia muerte con dignidad. Por otra parte, Jung también propuso una concepción culturalista de la sabldurla: el inconsciente colectivo; en las sociedades iletradas se conservarla en el folclore y en los cuentos de hades, en los que el arquetipo recurrente de hombre sabio (enciano con berba blanca y espesa) aparecía cuando la gente ee encontraba en situaclones desesperadas o ante problemas Insuperables (Holliday y Chandler, 1986).
} 
funcionamiento, crecimiento, supervivencia y adaptación humana. La función adaptativa de la sabiduria se asienta en las caracteristicas que generalmente se atribuyen a la sabiduria: procesos cognitivos (comprensión del mundo de forma desinteresada, búsqueda de las consecuencias últimas de los acontecimientos y la preservación de la integración del conocimiento), guia para la acción (virtud, valores, ...) y recompensa intrínseca (bien personal gratificante por si mismo). En esta misma línea, se ha querido ver a una persona sabia como aquélla que ha aprendido a equilibrar las tendencias opuestas de los aspectos cognitivos, afectivos y conativos de su comportamiento (Birren y Fisher, 1990).

Desde los primeros estudios cientficos, se considera que la sabiduría está compuesta por diversas características positivas como: habilidades interpersonales, madurez personal, integridad del yo, etc. (Clayton y Birren, 1980). Por tanto, ya desde sus comienzos, los sistemas de evaluación se enmarcan dentro del movimiento de psicología positiva que acentúa especialmente los aspectos positivos, cuando recoge datos sobre el funcionamiento humano (Lopez, Snyder y Rasmussen, 2003).

No abundan los procedimientos de evaluación psicológica de la sabiduria, y los que existen suelen estar ligados a las concepciones téricas sobre sabiduria que tengan sus autores. Los tres enfoques que consideramos más relevantes en la actualidad son:

(i) El Grupo de Berlín, que ha tenido una larga tradición, en el Instituto Max Plank, bajo el liderazgo de P. Baltes ${ }^{2}$, que considera la sabiduria como un conocimiento experto en la pragmática de la vida (Baltes, 1991, 1993). La sabiduria se operacionaliza sobre la base de cinco criterios: conocimiento fáctico profundo sobre la realidad de la vida; conocimiento procedimental acerca de cómo suceden las cosas; interpretación contextualizada del ciclo vital; relativismo en los valores; y conocimiento acerca de las incertidumbres y ambigüedades de la vida y su manejo. En cuanto a la evaluación de la sabiduria, emplean el pensamiento en voz alta: los sujetos verbalizan sus pensamientos (que se graban) mlentras aportan solución a 1 o 2 problemas de la

\footnotetext{
${ }^{2}$ Desgraciadamente, Paul Baltes falleció el 7 de noviembre del pasado año, lo que representa una sensible pérdida para la pslcologla en general, y sobre todo para la gerontologla, la psicologia del desarrolo del ciclo vital, y, por supuesto, para el estudio de la sabiduria desde la psicologia cientifica.
} 
vida que son propuestos por el evaluador; los protocolos son luego calificados, en cada uno de los cinco criterios, por calificadores entrenados.

(ii) La propuesta de Sternberg (1998) sobre la sabiduría, o teoría del equilibrio, define la sabiduría como la aplicación del conocimiento tácito, mediado por valores, dirigido hacia el logro del bien común a través de un equilibrio entre intereses intrapersonales, interpersonales y extrapersonales. En cuanto a la evaluación, este autor no presenta instrumentos específicos para medir su concepto de sabiduría. Aunque ha propuesto procedimientos para evaluar el conocimiento tácito dentro de su propuesta de inteligencia práctica (Sternberg et al., 1995), no está claro cómo se podría evaluar este conocimiento tácito dentro de su enfoque de sabiduria.

(iii) M. Ardelt (2004), basándose en los resultados empíricos sobre teorias implícitas de la sabiduría, llevados a cabo por Clayton y Birren (1980), considera que la sabiduría está compuesta por caracteristicas de personalidad que integra (a) aspectos cognitivos, (b) reflexivos y (c) afectivos.

a) La dimensión cognitiva de la sabiduría incluye: comprensión de la vida y deseo de conocer la verdad, conocimiento aceptación de aspectos positivos y negativos en la naturaleza humana, aceptación de los límites del conocimiento y de la incertidumbre en la vida.

b) La dimensión reflexiva referida a la capacidad de percibir los fenómenos y eventos desde distintas perspectivas, capacidad de autoanálisis, auto-comprensión y la superación de la subjetividad y las proyecciones.

c) La dimensión afectiva que incluye la empatia, la compasión y el amor por los semejantes.

Esta autora, a diferencia del Grupo de Berlín que se centra en el conocimiento relacionado con la sabiduria ante problemas hipotéticos propuestos por el examinador, hace más hincapié en lo que la persona es (en qué medida posee atributos de personalidad propios de persona sabia) y en su comportamiento en la vida, que en lo que la persona sabe. Propone, para medir la sabiduría, una escala autoaplicada de tres dimensiones, basada en la operacionalización de las tres dimensiones mencionadas anteriormente (cognitiva, reflexiva y afectiva). La versión final de la escala contiene 14 ítems en la 
dimensión cognitiva, 12 ítems en la reflexiva y 13 en la afectiva (Ardelt, 2003).

Aunque el interés por el estudio de la sabidurla en principio estaba centrado en poblaciones de personas adultas y ancianas, cada vez hay más estudios que se inte-resan por conocer la gestación y el crecimiento de las características relacionadas con la sabi-duría. La adolescencia es el periodo evolutivo en el que se originan algunas de las facetas que componen la sabiduria ( $p$. ej. razonamiento moral, toma de perspectivas, etc.) (Pasupathi, Staudinger y Baltes, 2001; Staudinger y Leipold, 2003; Richardson y Pasupathi, 2005). Estudios realizados por el Grupo de Berlín muestran que, aunque el rendimiento en conocimiento relacionado con la sabidurla es menor en los adolescentes que en los adultos, en el caso de los adolescentes y jóvenes (aproximadamente hasta los 20 años), la edad está asociada positivamente al conocimiento relacionado con sabiduria, mientras en los adultos no; esto nos indicaría que este periodo de la evolución humana, como apuntan los teóricos del Grupo de Berlin, es un campo abonado para que germine la semilla de la sabiduría (Pasupathi, Staudinger y Baltes, 2001). En muestras de adolescentes, se ha encontrado que la relación entre sabiduría, entendida como conocimiento relacionado con sabidurla (evaluada con un cuestionario auto-aplicado) ${ }^{4}$, y el rendimiento académico está modulada por el género: en los chicos casi no hay relación, y en las chicas existe relación positiva y significativa (González Leandro y Pelechano, 2004).

En este trabajo presentamos datos de fiabilidad, entendida como consistencia interna, y de validez factorial, de una escala de calificación sobre sabiduría en adolescentes que cursan sus estudios en la ciudad de Rosario (República Argentina). La puntuación en sabiduría de cada

\footnotetext{
'Consideramos que el procedimiento de evaluación propuesto por esta autora no es totalmente coherente con su propuesta teórica sobre sabidurla, ya que, utilizando sólo autointormes nunca se podrá garantizar que lo que el sujeto dice que hará, o que suele realizar, coincida con lo que realmente hace.

4 El cuestionario de conocimlento relacionado con sabidurla contemporánea se ha denominado «Cuestionario de constructivismo con fuerza de voluntad y control personaln, está compuesto de 50 ltems, de 4 alternativas sobre el grado de verdad que el sujeto esigna a cada uno de los ftems (ejemplo de Items: «s crees que puedes lograr algo y te empleas mucho en ello lo lograrasn, an tu vida siempre puedes elegir; por eso tu vida es le suma de todas tus eleccioness).
} 
alumno se obtuvo de las escalas de calificación que cumplimentaron sus profesores, sobre la base del conocimiento que tenian de sus alumnos; por ello decimos que la escala mide acciones relacionadas con la sabidurla (se entiende que los profesores forman sus opiniones de los alumnos basados en la observación de su comportamiento); en este sentido, la escala tiene un enfoque más cercano a la propuesta de Ardelt que a la del Grupo de Berlin: se trata de medir lo que el sujeto hace (tal como es percibido por personas allegadas, como son sus profesores), más que lo que dice que puede hacer (como es el caso de autoinformes ante problemas hipotéticos).

\section{MÉTODO}

\section{Muestra}

La muestra estuvo compuesta por 164 adolescentes (54\% hombres), con una media de edad de 15,6 años (D. T. 1,4), a los que pertenecian las escalas de calificación sobre sabiduría cumplimentadas por los profesores-tutores ${ }^{5}$, y que cursaban sus estudios en dos centros de secundaria de la ciudad de Rosario (República Argentina) ${ }^{6}$.

La distribución de los adolescentes en los distintos cursos se puede ver en la Tabla 1.

Cada profesor-tutor, solo o en colaboración con otros profesores que enseñan también a su grupo de alumnos, cumplimentó los 15 Items de la escala para cada uno de sus alumnos, sef̂alando la frecuencia con la que habla observado el comportamiento en el/la alumno/a.

\footnotetext{
S Aunque 80 solicito a los profesores-tutores (19, repartidos en dos centros) que cumplimentaran las escalas a algo más de $\mathbf{3 0 0}$ adolescentes, por motivos diversos sólo se pudieron recoger 164 escales cumplimentadas (un poco más de la mited), to que represente una proporctón algo mayor a lo que suele ser habltual en estudios de este tipo.

- Agradecemos la colaboración por la dirección y profesorado de los centros educativos: Escuela de Enseñanza Media «San Juan Bautista de ta Sallen y Escuela Media eServando Bayos; donde se pasaron las escalas de calificación; y sobre todo, a los profesores que emplearon parte de su tiempo para cumplimentarlas.
} 
Tabla 1. Número de alumnos $(N)$ en cada nivel académico y total

\begin{tabular}{|c|c|}
\hline CURSO & $\mathbf{N}$ \\
\hline Octavo & 15 \\
\hline Noveno & 31 \\
\hline Primero Polimodal & 67 \\
\hline Segundo Polimodal & 16 \\
\hline Tercero Polimodal & 35 \\
\hline TOTAL & 164 \\
\hline
\end{tabular}

\section{Instrumento}

La Escala de calificación de Acciones relacionadas con Sabiduría en Adolescentes (EASA) fue elaborada por nosotros para ser cumplimentada por profesores. Después de generar 20 items que consideramos representativos de las opciones teóricas sobre sabiduría que hemos descrito anteriormente, se sometieron al analisis de la adecuación del contenido por parte de los autores y de un psicólogo clinico perteneciente a nuestro equipo de investigación, eliminándose cinco items y quedando la escala definitiva, por tanto, reducida a 15 Ítems (véase Cuadro 1).

El formato de la escala de calificación se compone de 2 hojas. En la primera se informa de los objetivos del trabajo y de cómo se tiene que cumplimentar la escala. En la segunda, se presenta la escala propiamente dicha para que el profesor marque una de las cuatro alternativas de respuesta (nunca, alguna vez, frecuentemente, siempre) en cada uno de los items, correspondiente a la frecuencia con la que había observado el comportamiento en el/la alumno/a. 


\section{Procedimiento}

Después de contactar con las direcciones de los centros educativos y obtener los permisos correspondientes para llevar a cabo el estudio? se tuvo un primer contacto con el profesorado donde se explicó los objetivos del estudio y cuál era la actividad para la que solicitaba su colaboración. El equipo de investigación se comprometio a aportar un informe adelantando algunos resultados iniciales; informe que se entregó puntuaimente.

Cuadro 1. Items de la Escala de calificación de Acciones relacionadas con Sabiduria en Adolescentes (EASA), clasificados según la opción teórica de la que se derivan los Items

Teorias legas sobre sabiduría (Clayton y Birren, 1980) y sabiduría
como caracteristicas de personalldad (Ardelt, 2003):
1) Escucha y tiene en cuenta los consejos de otras personas
2) Cuando tiene algún problema con amigos o compañeros, reflexiona y
se suele poner en el lugar de la otra persona
3) Cuando le preocupa un problema, suele considerar distintas alternati-
vas posibles
4) Suele dividir a las personas en buenas o malas
5) Es más feliz ignorando aspectos de una situación que examinándola a
fondo
6) Se muestra compasivo cuando otras personas sufren por algo.
7) Comprende cómo se debe tratar a las personas y se siente bien cuan-
do está en compañía de gente
8) Trata de ayudar de alguna manera a los que lo necesitan

Sabiduría como conocimiento relacionado con sabiduría (Baltes, 1991, 1993):

9) En situaciones complejas de la vida diaria, muestra estrategias de conducta ajustadas y sensatas

10) Es una persona prudente

\footnotetext{
7 El presente trabajo es parte de un estudio más ampllo, del que es responsable el primer autor de este articulo, sobre teorlas legas respecto a la sabidurla y sobre componentes pslcologicos de la sabidurla, con perticipación de muestras de adolescentes canarios y argentinos.
} 
Cuadro 1. Items de la Escala de calificación de Acciones relacionadas con Sabiduria en Adolescentes (EASA), clasificados según la opción teórica de la que se derivan los items (continuación)

Sablduria como concepto relacionado con conocimiento tácito $y$, en general, con inteligencia práctica (Sternberg, 1998; Sternberg et al. 1995):

11) Es una persona intuitiva y perspicaz, es capaz de "leer entre líneas".

12) Saca partido a su experiencia y le gusta interesarse por los detalles para informarse mejor.

13) Es una persona con buen juicio en la mayoria de las ocasiones

14) Tiene facilidad para solucionar problemas, tanto escolares como sociales

15) Toma decisiones con facilidad hasta en situaciones poco claras

Cada profesor/a-tutor/a, solo/a o en colaboración con otros profesores que también impartian clase al grupo de alumnos, cumplimentaron la escala de calificación sobre sabiduria para cada uno de los/as alumnos/as. Una vez pasado el tiempo que se habla acordado para cumplimentar los protocolos (2 meses), un miembro del equipo de investigación pasó a recogerlos.

Al revisar los protocolos cumplimentados, el equipo investigador observó que, a pesar de haber hecho hincapié, verbalmente y por escrito, acerca de la importancia de no dejar ningún ítem sin contestar, en algunos protocolos apareclan items sin contestar. Se adoptó el criterio de cubrir este valor perdido con el valor medio en el item.

Todos los cálculos estadísticos realizados fueron llevados a cabo con el paquete estadistico SPSS, v. 14.

\section{RESULTADOS}

A) Estudio correlacional inter-ftems

En un primer análisis de los ítems, hemos realizado un estudio correlacional, cuyos resultados se muestran en la Tabla 2. En general, 


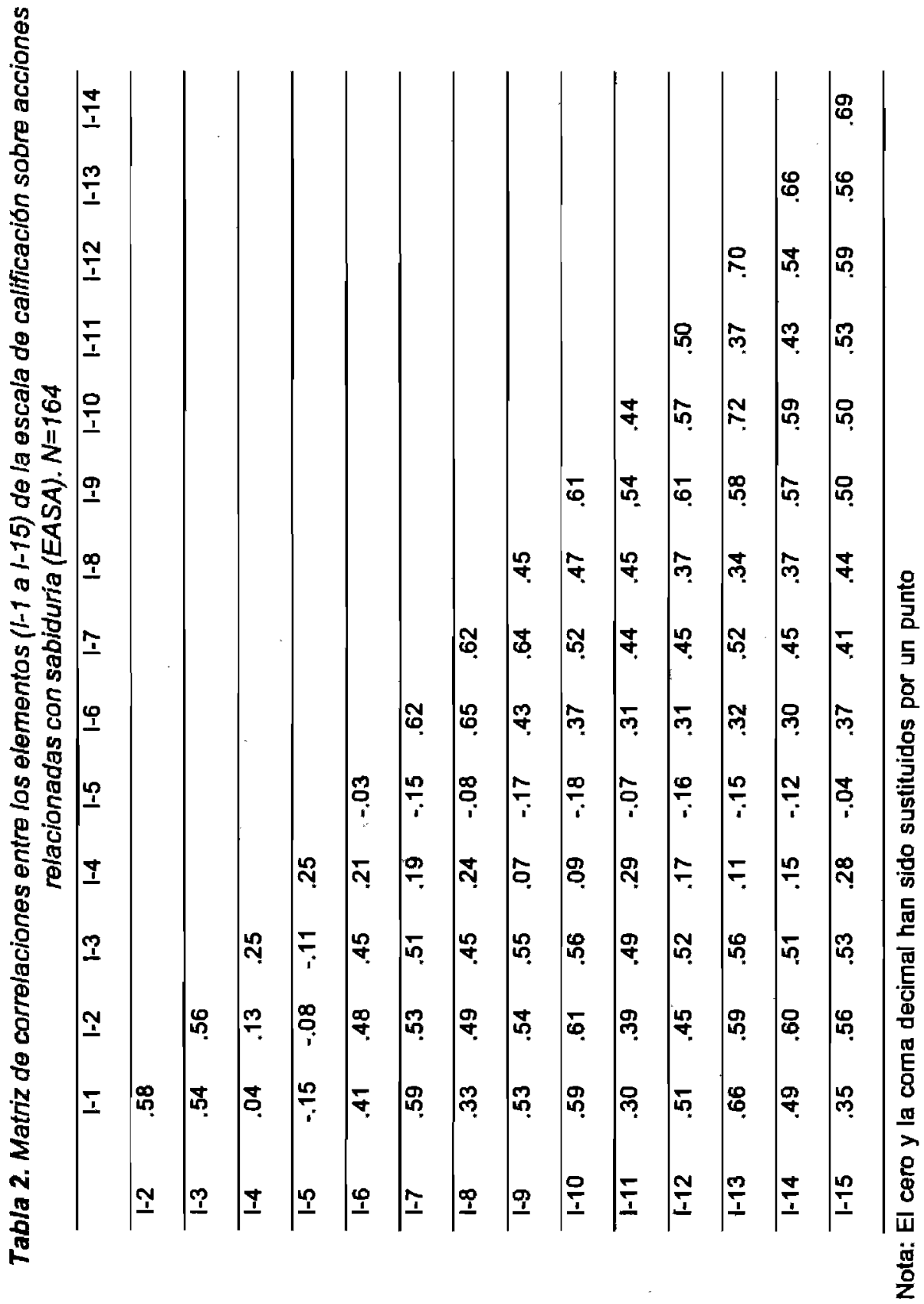


los elementos que componen la escala correlacionan entre si de forma significativa, excepto los Items 4 y 5 , que tienen correlaciones nulas o poco significativas, tanto entre sl como con el resto de Items. Debido a su comportamiento anómalo los hemos eliminado de la escala para posteriores análisis; la inadecuación de estos ítems puede haberse producido por problemas tanto en el formato de la pregunta como en el referente.

\section{B) Estructura factorial}

Los datos procedentes de las calificaciones asignadas por los profesores a cada uno de los Items (excepto los Items 4 y 5) fueron sometidos a un análisis factorial, por el método de componentes principales, y realizando luego una rotación oblicua, método normalización oblimín con Kaiser. Se obtienen 2 factores con valor propio mayor a 1 , que explican el $64 \%$ de la varianza $(54,5 \%$ el primer factor y $9,5 \%$ el segundo). La relación de items con las saturaciones mayores de 0,45 en cada factor se muestran en la Tabla 3.

Tabla 3. Resultados del análisis factorial con rotación oblicua. Matriz de configuración: saturaciones factoriales (mayores de 0,35) de los ftems en los dos factores (F-I y F-II) con asignación de cada variable a un solo factor. $N=164$

\begin{tabular}{|c|c|}
\hline ITEMS & F-I F-II \\
\hline 13. Es una persona con buen juicio... & .96 \\
\hline 14. Tiene facilidad para solucionar problemas... & .88 \\
\hline 12. Saca partido a su experiencia y le gusta ... & .86 \\
\hline 10. Es una persona prudente & .78 \\
\hline 15. Toma decisiones con facilidad... & .75 \\
\hline 9. En situaciones complejas de la vida diaria ... & .66 \\
\hline 1. Escucha y tiene en cuenta los consejos... & .65 \\
\hline 2. Cuando tiene algún problema con amigos... & .60 \\
\hline
\end{tabular}


Tabla 3. Resultados del análisis factorial con rotación oblicua. Matriz de configuración: saturaciones factoriales (mayores de 0,35) de los items en los dos factores (F-I y F-II) con asignación de cada variable a un solo factor. $N=164$ (continuación)

\begin{tabular}{lll}
\hline TTEMS & F-I & F-II \\
\hline
\end{tabular}

3. Cuando le preocupa un problema, suele ... $\quad .60$

11. Es una persona intuitiva y perspicaz... $\quad .49$

6. Se muestra compasivo cuando otras personas... $\quad .91$

8. Trata de ayudar de alguna manera a los que ...

7. Comprende cómo se dabe tratar a las personas... .66

Nota: El cero y la coma decimal han sido sustituidos por un punto

El primer factor, que denominamos «Sensatez, prudencia, pensamiento alternativo y capacldad para resolver problemas impersonales, personales e interpersonales», es un potente factor que tiene pesos factoriales elevados positivos en 10 Items.

El segundo factor, que denominamos «Compasion por los problemas del projimo y actitud de ayuda», tiene pesos factoriales positivos en 3 items.

En las Tablas 4 y 5 se muestra la matriz de estructura y la matriz de correlaciones entre los dos factores, respectivamente, y en ellas se aprecia que los factores no son totalmente independientes, observándose una relación moderada positiva. Podemos decir, por tanto, que la escala mide dos dimensiones o facetas de la sabidurla que están bastante relacionadas.

\section{C) Flabilidad}

El estudio de la fiabilidad de la escala de calificación EASA se llevó a cabo calculando la consistencia interna (alfa de Cronbach) de cada uno de los factores. Los resultados se muestran en la Tabla 6.

Los dos factores tienen coeficientes de consistencia interna superiores a 0,80, El coeficiente de fiabilidad (Alfa de Cronbach) del factor 1 es de 0,92, lo que indica una alta consistencia interna de sus items; y el del factor $2,0,84$. 
Se puede concluir que la fiabilidad de los dos factores de la escala EASA es muy alta.

Tabla 4. Resultados del análisis factorial con rotación oblicua. Matriz de estructura: saturaciones factoriales (mayores de 0,35 ) de los ltems en los dos factores ( $F-/ y$ - $-I I)$. $N=164$

\begin{tabular}{llll}
\hline \multicolumn{1}{c}{ ITEMS } & F-I & F-II \\
\hline 13. Es una persona con buen juicio... & .87 & \\
\hline 14. Tiene facilidad para solucionar problemas... & .81 & \\
\hline 10. Es una persona prudente & .81 & .47 \\
\hline 12. Saca partido a su experiencia y le gusta ... & .80 & \\
\hline 9. En situaciones complejas de la vida diaria, ... & .77 & .56 \\
\hline 15. Toma decisiones con facilidad... & .76 & \\
\hline 2. Cuando tiene algún problema con amlgos... & .74 & .58 \\
\hline 3. Cuando le preocupa un problema, suele ... & .73 & .56 \\
\hline 1. Escucha y tiene en cuenta los consejos... & .72 & .47 \\
\hline 11. Es una persona intuitiva y perspicaz... & .61 & .48 \\
\hline 6. Se muestra compasivo cuando otras personas... & & .88 \\
\hline 8. Trata de ayudar de alguna manera a los que ... & .50 & .86 \\
\hline 7. Comprende como se debe tratar a las personas... & .64 & .81 \\
\hline
\end{tabular}

Nota: El cero y la coma decimal han sido sustituidos por un punto

Tabla 5. Correlación entre los factores (F-l,y F-II) de la escala de calificación de acciones relaclonadas con sabidurla (EASA). $N=164$

$$
\text { F-2 }
$$

F-1 0,53


Tabla 6. Consistencia interna (coeficiente Alpha de Cronbach) de los factores de la escala de calificación sobre acciones relacionadas con sabidurla. $(N=164)$

\begin{tabular}{ccc}
\hline Factores & Núm. de Items & Coef. Alpha \\
& & \\
\hline F-I & 10 & 0,92 \\
\hline F-II & 3 & 0,84 \\
\hline
\end{tabular}

\section{CONCLUSIONES Y DISCUSIÓN}

La escala de calificación sobre acciones relacionadas con sabiduría para adolescentes (EASA) que hemos estudiado en este trabajo ha sido elaborada basándonos en algunos marcadores del constructo de sabiduría, según las teorlas contemporáneas más relevantes. La escala presenta una estructura factorial compuesta de 2 factores no independientes, el primero de ellos relacionado con la prudencia, la sensatez, el pensamiento alternativo (poder imaginar dlstintas alternativas de encarar y de solucionar problemas), y la capacidad para dar solución a problemas personales, interpersonales e impersonales. Y el segundo, relacionado con la actitud y tendencia hacia la compasión y ayuda hacia el projimo.

Desde el punto de vista psicométrico, la escala, pensamos que reúne condiciones psicométricas de flabilidad y validez factorial adecuadas. Aunque no podemos asegurar que los elementos que la componen constituyan una muestra totalmente representativa del constructo sabidurla (constructo del que, por otra parte, tampoco existe un consenso total entre investigadores), si se puede considerar como una aproximación a la evaluación de la sabiduria a través de un heteroinforme (en este caso, escala de calificación cumplimentada por otros); es decir, no registrando lo que el sujeto dice que habitualmente hace o va a hacer, sino registrando lo que suele hacer, según personas allegadas a él. No podemos asegurar, por tanto, que 
estén adecuadamente representadas todas las facetas o características que componen el constructo sabidurla; en este sentido, debemos ser cautos y considerar la escala como una medida de algunas dimensiones relacionadas con sabiduria.

Por el lado metodológico, en algunos Items parece que el lenguaje usado no fue lo suficientemente claro (de hecho hubo que eliminar dos items) y, además, como tal escala de calificación, sabemos que pudo verse afectada por una serie de sesgos frecuentes en estos instrumentos, tales como: comprensión diferencial de los items, sesgos no conscientes, efecto de halo, $y$, sobre todo $(y$, en parte, consecuencia de esos sesgos), por problemas de fiabilldad interjueces, que han podido causar algún tipo de distorsión a las calificaciones realizadas (Aiken, 1996; Sears, 1936). SI bien intentamos adelantarnos a la aparición de algunos de estos sesgos animando a los profesores-tutores a que cumplimentaran las escalas en colaboración con otros profesores, para posteriores estudios seria deseable, aparte de obtener algún indicador de la fiabilidad inter-jueces, establecer estrategias dirigidas a aumentar este tipo de fiabilidad, con sesiones de entrenamiento para los profesores en técnicas de calificación precisa y objetiva, previas al cumplimiento de las escalas.

Respecto a la muestra utilizada, somos conscientes de que no era muy grande y, además, probablemente poco representativa (muestreo no aleatorio y restringldo a dos centros de enseñanza); lo que nos sugiere realizar estudios con muestras distintas, más representativas, que nos permitan confirmar nuestros resultados.

Los problemas que hemos enumerado no nos deben llevar a invalidar los resultados que hemos obtenido. Al contrario, queremos resaltar que con nuestro trabajo, aparte de estudiar un procedimiento de evaluación poco usado en la evaluación de la sablduria, y de mostrar que el instrumento elaborado tiene unas caracteristicas de bondad psicométrica adecuadas, en cierta medida, emprendemos un camino prometedor en la metodologla de evaluación de la sabidurla desde un punto de vista cientifico. Asi, pensamos que el instrumento que presentamos aqui puede tener utilidad, aplicado a adolescentes escolarizados, al menos para dos fines: como una medida criterial para validar empiricamente otros instrumentos que se elaboren para evaluar sabiduría, por ejemplo, a través de autoinformes (Cronbach y 
Meehl, 1955); y como una medida del comportamiento sabio en ambientes escolares, que podría ser utilizada para la verificación de la eficacia de programas dirigidos a promocionar la sabiduria. Los estudios futuros que se lleven a cabo con este instrumento deberían avanzar en dos vias: por un lado, en el perfeccionamiento del instrumento y en la objetividad de su aplicación; y por otro, en el planteamiento de estudios que profundicen en la operacionalización del constructo sabiduria y en la validez externa y utilidad de la propia escala.

\section{REFERENCIAS BIBLIOGRÁFICAS}

Aiken, L. R. (1996). Personality assessment. Methods and Practices (2nd Ed.). Seattle: Hogrefe \& Huber Publishers.

Ardelt, M. (2003). Development and empirical assessment of a threedimensional wisdom scale. Research on Aging, 25, 275-324.

Ardelt, M. (2004). Wisdom as Expert Knowledge System: A Critical Review of a Contemporary Operationalization of an Ancient Concept, Human Development, 47:257-285

Baltes, P.B. (1991). The many faces of human ageing: Toward a psychological culture of old age. Psychological Medicine, 21, 837854.

Baltes, P.B. (1993). The aging mind: Potential and limits. The Gerontologist, 33, 580-594.

Birren, J. E. y Fisher, L. M. (1990). Integration of approaches and viewpoints. En R. J. Sternberg (ed.). Wisdom: Its nature origins and development. Nueva York: Cambridge University Press..

Clayton, V. P. y Birren, J. E. (1980). The development of wisdom across the life-span: A re-examination of an acient topic. En P. C. Baltes y O. G. Brian (eds.). Life-span development and behaviour, vol. 3. Nueva York: Academic Press.

Cronbach, L. J. y Meehl, P. E. (1955). Construct validity in psychological tests, Psychological Bulletin, 52, 281-302.

Csikszentmihalyi, M. y Rathunde, K. (1990). The psychology of wisdom: An evolutionary interpretation. En R. J. Sternberg (ed.). Wisdom: Its nature origins and development. Nueva York: Cambridge University Press. 
Erikson, E. H. (1959). Identity and the life cycle. Nueva York: International University Press.

Erikson, E. H. (1968). Identity: Youth and crisis. New York: Norton.

Holliday, S. G. y Chandler, M. J. (1986). Wisdom: Expiorations in adult competence. Basel: Karger.

González Leandro, P. y Pelechano, V. (2004). Sabidurla contemporánea, motivación y rendimiento académico en adolescentes. Análisis y Modificación de Conducta, 30, 963-976.

Lopez, S. J., Snyder, C. R. y Rasmussen, H. N. (2003). Striking a vital balance: Developing a complementary focus on human weakness and strength through posirive psychological assessment. En S. J. Lopez y C. R. Zinder (Eds.). Positive Psychological Assessment. A Handbook of Models and Measures. Washington: American Psychological Association.

Pasupathi, M., Staudinger, U. M. y Baltes, P. B. (2001). Seeds of wisdom: Adolescents' knowledge and judgment about difficult life problems. Developmental Psychology, V. 37, 3, 351-361.

Sears, R. R. (1936). Experimental studies of projection: I. Attribution of traits. Joumal of Social Psychology, 7, 151-163.

Staudinger, U. M. y Leipold, B. (2003). The assessment of wisdomrelated performance. . En S. J. Lopez y C. R. Zinder (Eds.). Positive Psychological Assessment. A Handbook of Models and Measures. Washington: American Psychological Association.

Sternberg, R. (1998). A balance theory of wisdom. Review of General Psychology, V. 2, 4, 347-365.

Sternberg, R.J., Wagner, R.K., Williams, W.M., y Horvath, J.A. (1995).

Testing common sense. American Psychologist, 50, 912-927. 